\title{
EMCCD photometry reveals two new variable stars in the crowded central region of the globular cluster NGC 6981 (Corrigendum)
}

J. Skottfelt ${ }^{1,2}$, D. M. Bramich ${ }^{3}$, R. Figuera Jaimes ${ }^{3,4}$, U. G. Jørgensen ${ }^{1,2}$, N. Kains ${ }^{3}$, K. B. W Harpsøe $\mathrm{e}^{1,2}$, C. Liebig ${ }^{4}$, M. T. Penny ${ }^{5}$, K. A. Alsubai ${ }^{6}$, J. M. Andersen ${ }^{7,2}$, V. Bozza ${ }^{8,9}$, P. Browne ${ }^{4}$, S. Calchi Novati ${ }^{10}$, Y. Damerdji ${ }^{11}$, C. Diehl ${ }^{12,13}$, M. Dominik ${ }^{4, \star}$, A. Elyiv ${ }^{11,14}$, E. Giannini ${ }^{12}$, F. Hessman ${ }^{15}$, T. C. Hinse ${ }^{16}$, M. Hundertmark ${ }^{4}$, D. Juncher ${ }^{1,2}$, E. Kerins ${ }^{17}$, H. Korhonen ${ }^{1,2}$, L. Mancini ${ }^{18}$, R. Martin ${ }^{19}$, M. Rabus ${ }^{20}$, S. Rahvar ${ }^{21}$, G. Scarpetta ${ }^{10,8,9}$, J. Southworth ${ }^{22}$, C. Snodgrass ${ }^{23}$, R. A. Street ${ }^{24}$, J. Surdej ${ }^{11}$, J. Tregloan-Reed ${ }^{22}$, C. Vilela ${ }^{22}$, and A. Williams ${ }^{19}$

${ }^{1}$ Niels Bohr Institute, University of Copenhagen, Juliane Maries Vej 30, 2100 København Ø, Denmark e-mail: skottfelt@astro.ku.dk; uffegj@nbi.dk

2 Centre for Star and Planet Formation, Natural History Museum, University of Copenhagen, Østervoldgade 5-7, 1350 København K, Denmark

${ }^{3}$ European Southern Observatory, Karl-Schwarzschild-Straße 2, 85748 Garching bei München, Germany e-mail: dbramich@eso.org

${ }^{4}$ SUPA, University of St Andrews, School of Physics \& Astronomy, North Haugh, St Andrews, KY16 9SS, UK e-mail: rfiguera@eso.org; robertofiguera@gmail.com

5 Department of Astronomy, Ohio State University, 140 W. 18th Ave., Columbus, OH 43210, USA

6 Qatar Foundation, PO Box 5825 Doha, Qatar

Department of Astronomy, Boston University, 725 Commonwealth Avenue, Boston, MA 02215, USA

8 Dipartimento di Fisica "E. R. Caianiello", Università di Salerno, via Ponte Don Melillo, 84084 Fisciano (SA), Italy

9 Istituto Nazionale di Fisica Nucleare, Sezione di Napoli, Napoli, Italy

10 Istituto Internazionale per gli Alti Studi Scientifici (IIASS), 84019 Vietri Sul Mare (SA), Italy

${ }^{11}$ Institut d'Astrophysique et de Géophysique, Université de Liège, Allée du 6 Août, Bât. B5c, 4000 Liège, Belgium

12 Astronomisches Rechen-Institut, Zentrum für Astronomie der Universität Heidelberg, Mönchhofstr. 12-14, 69120 Heidelberg, Germany

13 Hamburger Sternwarte, Universität Hamburg, Gojenbergsweg 112, 21029 Hamburg, Germany

14 Main Astronomical Observatory, Academy of Sciences of Ukraine, vul. Akademika Zabolotnoho 27, 03680 Kyiv, Ukraine

15 Institut für Astrophysik, Georg-August-Universität Göttingen, Friedrich-Hund-Platz 1, 37077 Göttingen, Germany

16 Korea Astronomy and Space Science Institute, 305-348 Daejeon, Republic of Korea

17 Jodrell Bank Centre for Astrophysics, University of Manchester, Oxford Road, Manchester M13 9PL, UK

18 Max Planck Institute for Astronomy, Königstuhl 17, 69117 Heidelberg, Germany

19 Perth Observatory, Walnut Road, Bickley, Perth 6076, WA, Australia

20 Departamento de Astronomía y Astrofísica, Pontificia Universidad Católica de Chile, Av. Vicuña Mackenna 4860, 7820436 Macul, Santiago, Chile

21 Department of Physics, Sharif University of Technology, PO Box 11155-9161 Tehran, Iran

22 Astrophysics Group, Keele University, Staffordshire, ST5 5BG, UK

23 Max-Planck-Institute for Solar System Research, Max-Planck Str. 2, 37191 Katlenburg-Lindau, Germany

${ }^{24}$ Las Cumbres Observatory Global Telescope Network, 6740 Cortona Drive, Suite 102, Goleta, CA 93117, USA

A\&A 553, A111 (2013), DOI: 10.1051/0004-6361/201321430

Key words. globular clusters: individual: NGC 6981 - stars: variables: general - stars: variables: RR Lyrae instrumentation: high angular resolution - errata, addenda

The celestial coordinates of the two variable stars in Table 1 have unfortunately been transposed. The correct table is shown here.

\footnotetext{
^ Royal Society University Research Fellow.
} 
A\&A 558, C1 (2013)

Table 1. Details of the two new variable stars found in NGC 6981.

\begin{tabular}{lccccc}
\hline \hline $\begin{array}{l}\text { Variable } \\
\text { star ID }\end{array}$ & $\begin{array}{c}\text { Var. } \\
\text { type }\end{array}$ & $\begin{array}{c}\text { RA } \\
(\mathrm{J} 2000.0)\end{array}$ & $\begin{array}{c}\text { Dec } \\
(\mathrm{J} 2000.0)\end{array}$ & $\begin{array}{c}T_{\max } \\
(\mathrm{d})\end{array}$ & $\begin{array}{c}P \\
(\mathrm{~d})\end{array}$ \\
\hline V57 & $\mathrm{RR} 1$ & 205327.38 & -123213.3 & 6166.779 & 0.334 \\
V58 & $?^{a}$ & 205327.12 & -123213.9 & 6166.76 & 0.285 \\
\hline
\end{tabular}

Notes. The celestial coordinates correspond to the epoch of the reference image, which is the heliocentric Julian date $\sim 2456167 \mathrm{~d}$. The epoch of maximum light is given as a heliocentric Julian date $(2450000+)$ in Col. 5 and the period is given in Col. $6 .{ }^{(a)}$ We are unable to classify this variable. 